Textures and Microstructures, Vol. 34, pp. 147-157 Reprints available directly from the publisher Photocopying permitted by license only
(C) 2000 OPA (Overseas Publishers Association) N.V.

Published by license under the Gordon and Breach Science Publishers imprint. Printed in Malaysia.

\title{
TEXTURE AND MICROSTRUCTURE CONTROL IN ZINC COATED STEEL SHEETS. EFFECT ON FORMABILITY OF THE COATINGS
}

\author{
S. GAIGNARD ${ }^{a, *}$, S. LAZIK $^{a}$, J. LIETZAU ${ }^{a}$, J. WEGRIA $^{b}$, \\ M. DUBOIS ${ }^{c}$, M.J. PHILIPPE ${ }^{a}$ and C. ESLING ${ }^{a}$ \\ a LETAM URA CNRS, 2090 Metz University, BP 80794, F 57012 Metz

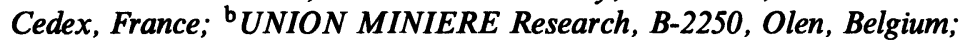 \\ ${ }^{\circ}$ COCKERILL SAMBRE, B-4400, Flemalle, Belgium
}

(Received 8 October 1999)

Many commercial galvanised steel sheets have been characterised before deformation (grain size, grain distribution in the coatings, pre-cracking, individual orientations, misorientations between neighbouring grains, local and global textures) in connection with the zinc alloy which was used and the process parameters.

The samples were bent by a biaxial expansion and examined afterwards. All the coatings were pre-cracked before deformation.

The extent of the pre-cracking depends on the intensity and the sharpness of the basal planes texture of the coating and also on the misorientations across the grain boundaries. In the same way, zinc primary dendrites and recrystallised grains can hinder the crack propagation, limiting their extent.

We can establish a correlation between the texture, the microstructure of the coatings (using a concept that corresponds to the mean free path of the crack) and the formability. As we know the process parameters, it is now conceivable that the texture of the coatings could be controlled.

Keywords: Hot-dip zinc; Microstructure; Texture; EBSD

\section{INTRODUCTION}

Galvanising steel sheet is an economic and effective process to protect steel against corrosion. During forming operations of coated steels, for

\footnotetext{
* Corresponding author.
} 
example stamping, cracking can occur and decrease the efficiency of galvanisation. The texture is the most important parameter as far as the anisotropy of the material properties is concerned (Nowak and Oles, 1990). Its influence is mainly important on the deformation behaviour parameters. This work focuses on the metallographic preparation. We present texture determination by $\mathrm{X}$-ray goniometer and by EBSD. First, the correlation between the microstructure, the texture and the deformation behaviour of the zinc coating was studied on 80 samples. Four characteristic samples were particularly examined. Second, we have looked for the optimal process parameters that allow to control the texture of the coatings.

Due to the $c / a$ ratio, basal slip is the predominant deformation mode in zinc (Patridge, 1967). The angle of the basal planes in relation with the deformation axis and, consequently, with the surface of the steel substrate determines to a large extent the deformation behaviour of the zinc coating.

Thus it is obvious that the microstructure and the texture will influence the mechanical behaviour of the coating. We first explain clearly the experimental process that permits the determination of the texture of the zinc. Second, we show relations between the texture, the microstructure and the deformation behaviour with some samples having different compositions and being produced with different process parameters, to obtain the optimal texture and microstructure and thus to obtain the best deformation behaviour.

\section{EXPERIMENTAL PROCEDURE}

\section{Materials}

In order to establish correlation between the texture, the microstructure and the deformation behaviour, more than 80 hot-dip galvanised steel sheets (industrially produced by Cockerill-Sambre) were studied (Lazik et al., 1996). The difference between these samples comes from the chemical composition $(\mathrm{Pb}, \mathrm{Al})$, process parameters, mechanical treatment and thickness of the coating. The samples used are detailed in Table I. They corresponded to specific cases which were particularly interesting (Lietzau et al., 1998). It is obvious that we have to resort to a statistically relevant method. 
TABLE I Details of the process parameters for the studied samples

\begin{tabular}{lcccc}
\hline & \multicolumn{4}{c}{ Sample } \\
\cline { 2 - 5 } & 1 & 2 & 3 & 4 \\
\hline Al-bath (\%) & 0.16 & 4.71 & 0.35 & 0.2 \\
Pb-bath (\%) & 0.12 & 0 & 0.005 & 0.07 \\
$T$-bath $\left({ }^{\circ} \mathrm{C}\right)$ & 485 & 434 & 460 & 470 \\
Skin-pass (\%) & 0 & 0.64 & 1 & $?$ \\
Al-coating (\%) & 0.86 & 4.81 & 0.44 & 0.29 \\
Pb-coating (\%) & 0.14 & 0 & 0 & 0.08 \\
Coating weight $\left(\mathrm{g} / \mathrm{m}^{2}\right)$ & 226 & 263 & 282 & 250 \\
$\quad$ on two faces & & & & \\
\hline
\end{tabular}

In all the cases, the substrate was a low carbon steel, and the thickness of the coating was about $20 \mu \mathrm{m}$.

\section{Experimental Work}

In order to determine a sample's texture, three methods are possible: pole figure measurement with a texture goniometer, EBDS analysis and determination of individual grain orientation with a X-ray diffractometer.

The texture measurements were carried out with an automatic X-ray texture goniometer. In the present study, the main texture difference between the various samples is due to the tilt of the $c$-axes. So, we just measured (00.2) incomplete pole figures. These pole figures were measured by $\mathrm{X}$-ray diffraction up to $78.5^{\circ}$ tilt angle on the surface of the zinc coatings, after cutting and cleaning according to the same conditions for all the samples. In this range of angle no defocalisation or line broadening correction was needed (Patridge, 1967). The radiation used was $\mathrm{Co}-\mathrm{K} \alpha$, at $40 \mathrm{kV}$ and $25 \mathrm{~mA}$. The pole figures were measured with angular steps of $\Delta \chi=2.5^{\circ}$ for the tilt angle and $\Delta \varphi=5^{\circ}$ for the azimuthal angle. The pole figure densities were pseudo-normalised i.e., only the measured areas $\left(0^{\circ}<\chi<78.5^{\circ}\right)$ have been considered in the normalisation procedure.

For the study of crystallographic texture, a huge number of individual grain orientations had to be determined. In order to have a satisfactory statistical relevance, 1000 grains at least were required. In order to make comparisons between different pole figures we had to make sure that they were always based on a similar number of grains. When the number of crystallites contributing to the diffraction was too small, the 
statistics was improved by the superimposition of several measured pole figures.

The goniometer, equipped with a laser beam, allows to point a specific zone (like grains larger than $500 \mu \mathrm{m}$ ), we could then find orientation of each selected grain manually, which was rather time consuming.

The measurement of individual grain orientation, in particular the "automated Kikuchi technique" with Transmission Electron Microscopes (TEM) as well as with Scanning Electron Microscopes (SEM) has been developed to a high standard (Schwarzer, 1997). Kikuchi patterns did not find a wide application in zinc because of several experimental difficulties (it was difficult to obtain good Kikuchi patterns by using backscattered electrons). The oxide layer was easily removed by a proper etching and the problem arose if there were internal strains in nonrecrystallized samples. Electron Back Scattering Diffraction (EBSD) allows the measurement of the individual orientation of each grain with a SEM. Now, it is possible to have a good image with materials like zinc, provided there is a very careful sample preparation. This method is particularly convenient to delimit the domain's size. The notion of domains corresponds to the free mean path of cracks (Fig. 1).
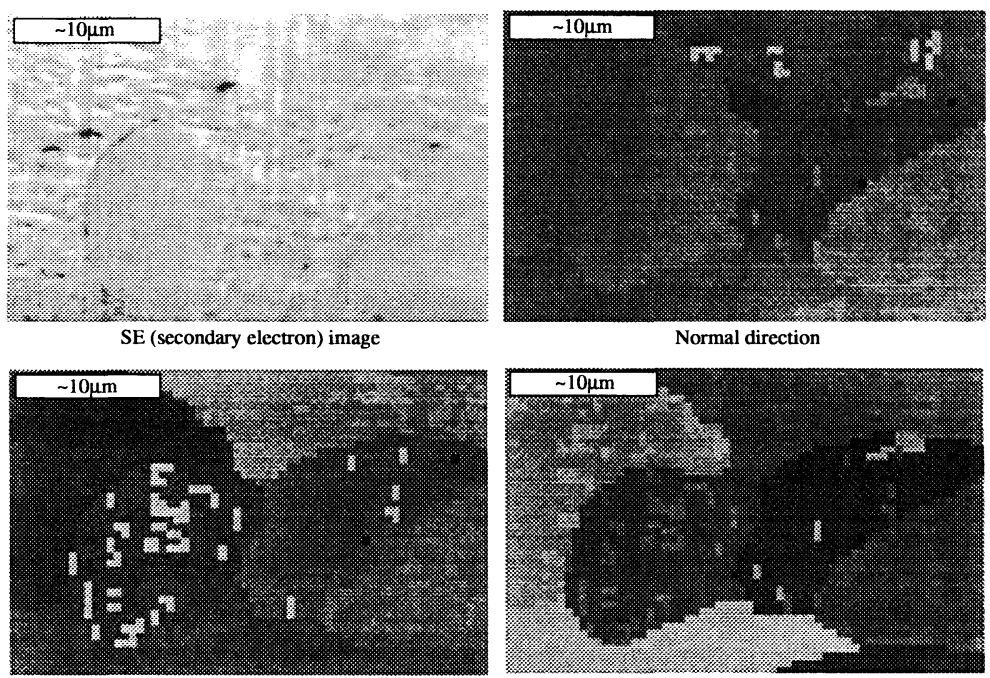

Transverse direction

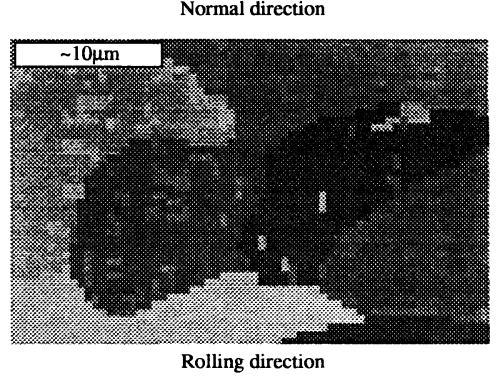

FIGURE 1 EBSD mapping of Galfan. 
The coated surfaces of the non-deformed samples were prepared metallographically (mechanical polishing and chemical etching) and observed with an optical microscope (Zeiss Axiophot). In addition, some samples were observed with a SEM (Leica S440, Hitachi S2500) too.

The coating cracking behaviour was determined by bending tests and biaxial expansion. All these tests were carried out in the R\&D centre of Cockerill-Sambre, Belgium.

Pre-cracking prior to the deformation was found and the evolution of these cracks with the deformation has been observed.

\section{RESULT AND DISCUSSION}

Sample 1 This sample has a strong texture. The (00.2) pole figure shows several single peaks with very high intensities. The grain size is $1400 \mu \mathrm{m}$. Each single peak in this pole figure can be related to the diffraction of one grain. Almost all the grain boundaries show cracking (Figs. 2 and 3).

Sample 2 The (00.2) pole figure presents no very strong basal texture. Spangle size is almost $700 \mu \mathrm{m}$ but an EBSD analysis shows a good cartography with coherent orientation zones, which are smaller (dendrites and eutectics zones have different orientations). The size of

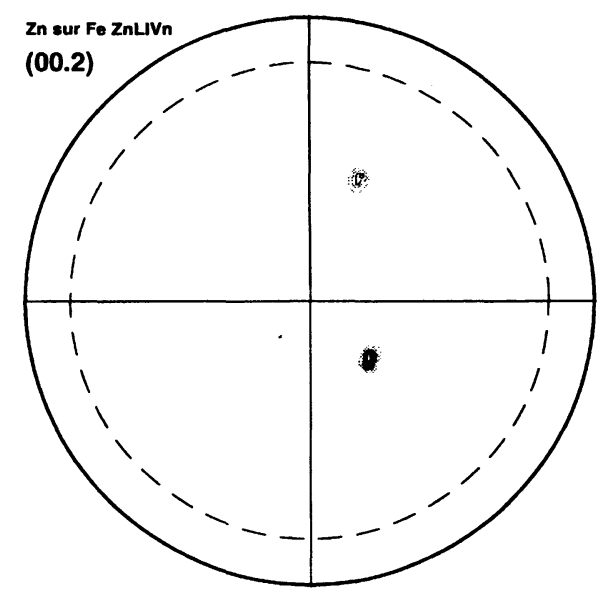

FIGURE 2 (00.2) pole figure of sample 1: several single peaks. 


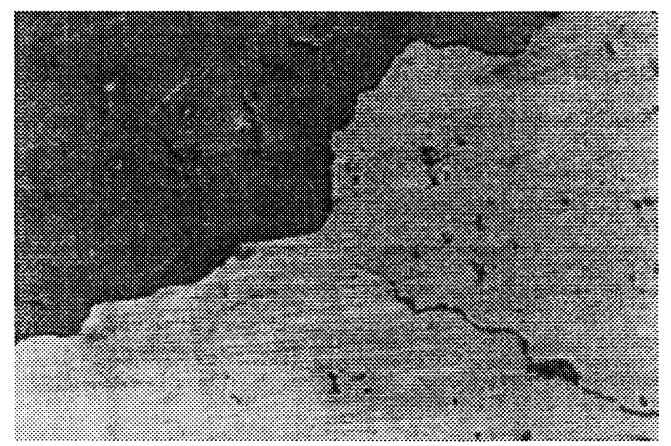

FIGURE 3 Sample 1, 100×, microstructure before deformation.

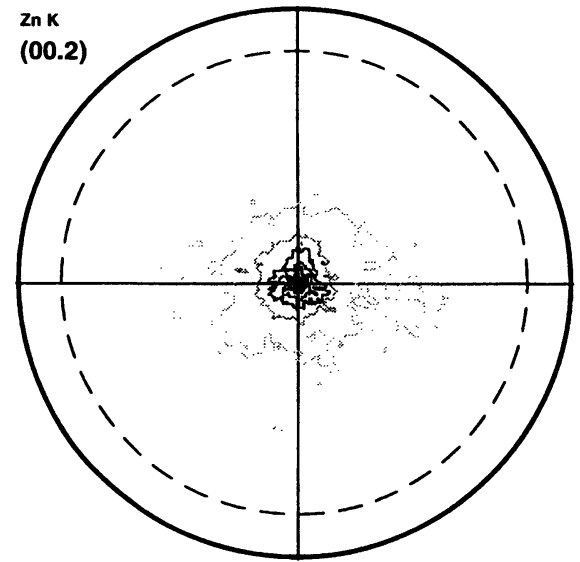

FIGURE $4(00.2)$ pole figure of sample 2: no strong basal texture.

coherent domains is $110 \mu \mathrm{m}$ in reality. The grain boundaries are intermittently cracked (Figs. 4 and 5).

Sample 3 The texture of this sample is a fairly strong basal texture (Fig. 6). Microstructure shows grains of $500 \mu \mathrm{m}$ size that contain twins or new recrystallised grains (Fig. 7). EBSD shows that the size of the domains with coherent orientation is about $100 \mu \mathrm{m}$ diameter. The boundary cracks are much more rare.

Sample 4 This sample has a very sharp basal texture (Fig. 8). Grain size is measured to be $100 \mu \mathrm{m}$ using an optical microscope (Fig. 9) but, in reality, a lot of neighbouring grains have the same 


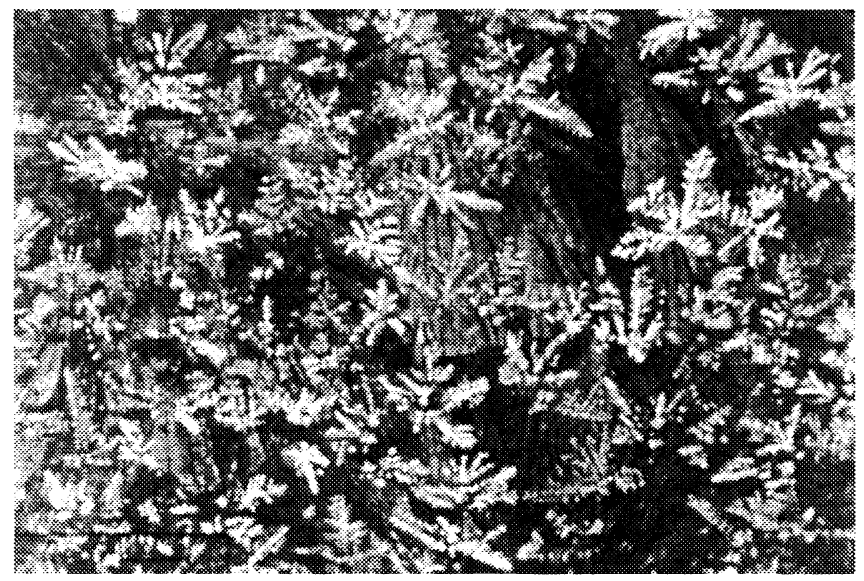

FIGURE 5 Sample 2, 100x, primary zinc dendrites in Galfan coatings.

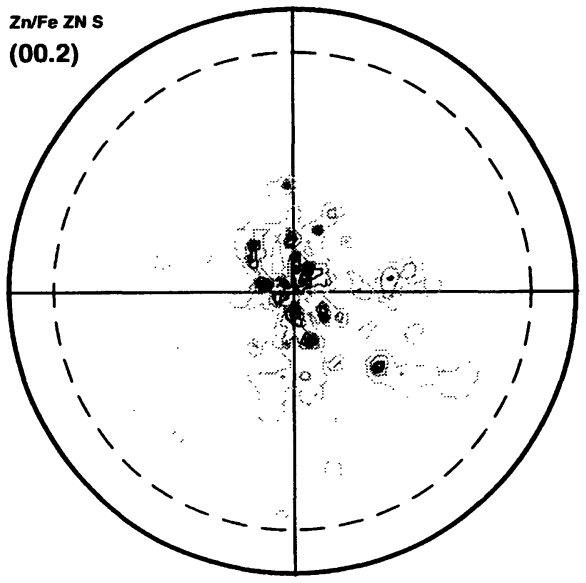

FIGURE $6(00.2)$ pole figure of sample 3: fairly strong texture.

orientation and so, the size of the crystallographically coherent domains is much more larger $(\gg 1000 \mu \mathrm{m})$. Almost all the grain boundaries of this sample show cracks.

The differences between the samples appeared more clearly when observed at large deformation $(\varepsilon=0.13)$. Among the four studied coatings, the sample 4 can be considered as the worst. In fact, the deformation was accommodated by the enlargement of the cracks along 


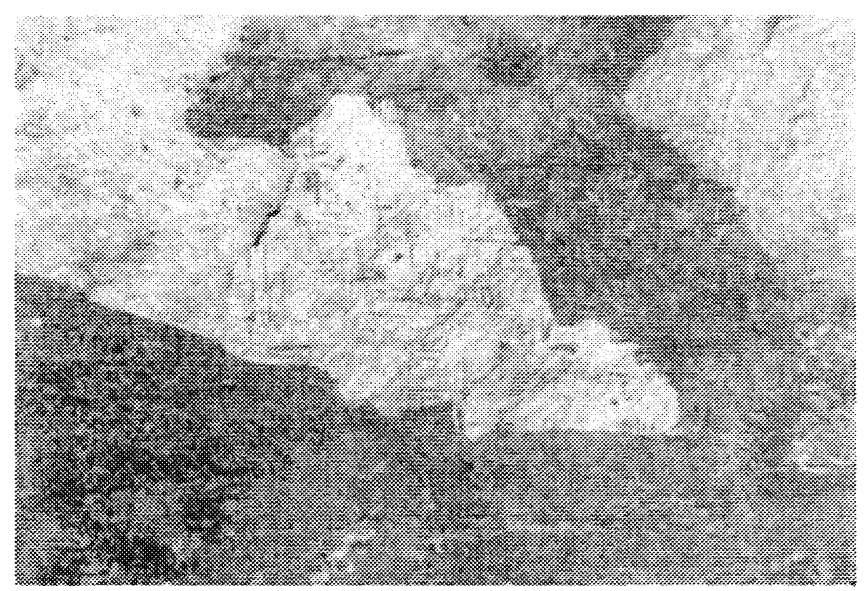

FIGURE 7 Sample 3, 100×, microstructure before deformation.

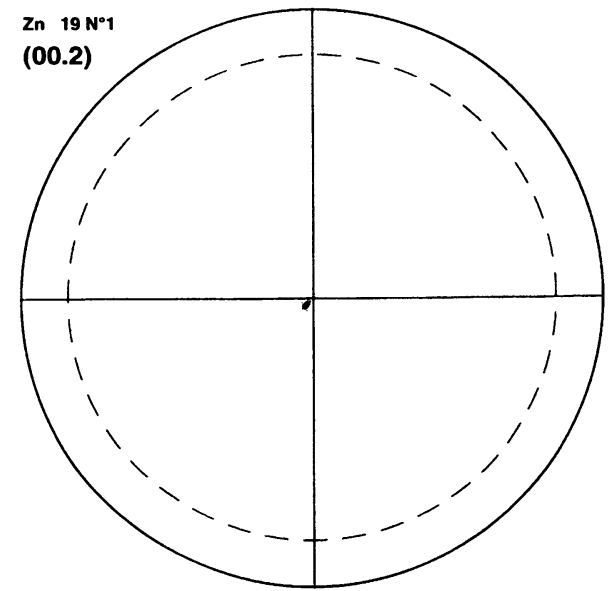

FIGURE $8(00.2)$ pole figure of sample 4: this sample presents a very sharp basal texture.

the grain boundaries. The texture of the coating of this sample is very sharp, the basal planes being parallel to the substrate surface. As the grains were already twinned before the deformation, there was no further possibility to accommodate the imposed deformation. In sample 1, grain boundary cracks as well as cleavage in some grains could be observed in the early stage of the deformation, from deformations as 


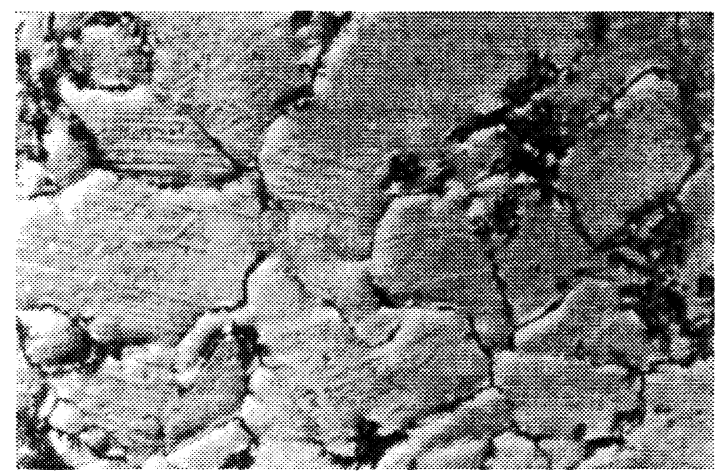

FIGURE 9 Sample 4, 200×, microstructure before deformation.

small as $2 \cdot 10^{-2}$. Sample 3 simultaneously presents areas which could accommodate the deformation, delimited by smooth grain boundaries without cracks and undeformed grains that were partially separated from the substrate (Deits and Matlock, 1990). As there was no particular obstacle, no impurities segregation at the grain boundaries, the microstructural features had to be related to the orientation of the grains and to the grain boundaries. The microstructure of the sample 2 shows that there is no continuous cracking, even at large deformations. Cracks formed inside the eutectic colonies were observed, but they had no influence on the cracking resistance (Lietzau et al., 1998).

\section{DISCUSSION AND CONCLUSIONS}

More than 80 industrial samples were analysed, and they were characterised by:

- various chemical compositions (contents in $\mathrm{Pb}, \mathrm{Al}, \mathrm{Si}$ );

- various galvanising conditions;

- various mechanical treatment after galvanisation.

All the coatings presented pre-cracks before the deformation. These cracks certainly arose from the thermal stresses due to the difference between the thermal expansion coefficients of steel and zinc (respectively: $12 \cdot 10^{-6}$ and $26 \cdot 10^{-6} \mathrm{~K}^{-1}$ ) (Jaffrey et al., 1980). These cracks were located at grain boundaries because during solidification, zinc 
contracts for $3 \%$ and so the thickness mainly decreases at the grain boundaries (the last solidified areas). The extent of the pre-cracking was depending on both the intensity and the sharpness on the basal texture of the coating and also on the size of the domains. The sharpness of the peaks varied according to the galvanisation process.

When all the grains had a strong basal orientation, an extensive cracking was observed after the bending test. In this case, there was no slip between the grains, the activation of the basal slip system (00.1) $\langle 11.0\rangle$ was not possible because the resolved shear stress was too low. The only possibility to accommodate deformation is cleavage. As several neighbouring grains had the same orientation, the crack propagation was not hindered. These samples had a much larger domain size than their grain size.

During the deformation, the cracks were enlarging and propagating along the grain boundaries. Zinc primary dendrites delimited the size of the domains and could hinder the propagation of cracks. In Al-rich alloys, the domains corresponded with regions with proeutectic or eutectic precipitation, depending on the $\mathrm{Al}$ content. The eutectic alloy Galfan with about $5 \% \mathrm{Al}$, demonstrated a very good cracking behaviour, as an extensive surface deformation without cracks could be observed after the bending test. This may also have been due to the presence of the fcc solid solution of aluminium in zinc, which supplies additional slip systems. The small size of the domains is probably the main reason for the enhancement of cracking resistance.

In the case of a weak basal texture, only a few crystallites had their $c$-axis oriented parallel to the substrate normal. There were crystallites with a relatively important tilt of the $c$-axis versus the normal direction to the substrate surface. The slip systems were preferentially active in these crystals and consequently, the tilted grains could accommodate the plastic deformation. However, crystallites having their $c$-axis normal to the substrate surface, remained undeformed. In these samples, the size of the domains were of the order of the grain size, because the crystallites had quite a different orientation from one to its neighbours. Sometimes, the domain size was even smaller than the grain size, because small recrystallised grains had grown in the limits of former crystallites.

If the sample had a strong texture with basal planes parallel to the substrate surface, it would be difficult to accommodate deformation by gliding (Schmid factor too small), all the more when the grains were 
twinned prior to the deformation (Fig. 9). Individual grain orientations can be separately distinguished in the pole figure, which indicates that the statistics of grains is rather poor. The cracking resistance may not be sufficient for these samples.

A clear correlation between the texture, the microstructure and the cracking resistance has been established in the present study. Higher the density of subgrains, recrystallised grains and twins is, more the size of the domains decrease, weaker the intensity of the $(00.2)$ pole figure is, better the cracking resistance will be. The sharpness of the $c$-axes peaks and its tilt angle in relation to the substrate normal determine the deformation behaviour of the coating layer. Zinc primary dendrites and recrystallised grains can hinder the crack propagation. This means that process parameters that enable the appearance of nuclei of recrystallised and zinc primary dendrites can improve the resistance to propagation of cracks in zinc coatings. We thus chose to test as process parameters the skin-pass reductions to create recrystallised grains, and the cooling rates, to make zinc primary dendrites appear.

\section{References}

Deits, S.H. and Matlock, D.K.: Formability of coated sheet steels: An analysis of surface damage mechanisms. In Zinc-Based Steel Coating Systems, G. Krauss and D.K. Matlock (Eds.) (Warrendale, PA: Metallurgy and Performance, TMS, 1990), pp. 297-318.

Jaffrey, D., Browne, J.D. and Howard, T.J.: The cracking of zinc spangles on hot-dipped galvanized steel. Met. Trans. B, 11B (1980), 631-635.

Lazik, S., Esling, C., Philippe, M.J., Wégria, J. and Dubois, M.: A metallurgical approach to the cracking resistance of hot-dip galvanised zinc coatings, Texture and Microstructure, 28 (1996), 35-45.

Lietzau, J., Philippe, M.J., Esling, C., Wégria, J. and Dubois, M.: Pre-cracking of zinc coated steel sheets during deformation, Zinc-Based Steel Coating Systems (1998), 207-217.

Nowak, R. and Oles, A.: Anisotropy of failure of a hexagonal metal with preferred crystallite orientation. Yielding, Damage and Failure of Anisotropy Solids, EGFS, J.P. Boehler (Ed.), Mechanical Engineering Publication, London, 1990, pp. 747-765.

Patridge, P.G.: The crystallography and deformation modes of hexagonal close-packed metals, Metallurgical Reviews, Review 118 (1967), 169-194.

Schwarzer, R.: Automated crystal lattice orientation mapping using a computer-controlled SEM, Micron, 28 (1997), 249-265. 